\title{
A strain-rate dependent engineering approximation of the temperature rise in plastically-deforming DP steel
}

\author{
Xueyang Li, Christian C. Roth*, and Dirk Mohr \\ Chair of Computational Modelling of Materials in Manufacturing, Department of Mechanical and \\ Process Engineering, Swiss Federal Institute of Technology (ETH), Zurich, Switzerland
}

\begin{abstract}
Experiments at ten strain rates ranging from $0.001 / \mathrm{s}$ to $4 / \mathrm{s}$ are carried out on uniaxial tension specimens extracted from DP800 metal sheets. Digital Image Correlation (DIC) is used to obtain surface strain fields and a high speed infrared (IR) camera is employed to measure the corresponding temperature rise due to plastic dissipation. A temperature rise of $60 \mathrm{~K}$ is witnessed for the highest loading speed whereas minimal temperature rise $(<1 \mathrm{~K})$ is seen for the lowest loading speed. To minimize the computational cost by treating the temperature as an internal state variable, (effectively avoiding more complex coupled thermo-mechanical analyses), a logarithm based function is proposed that models the transition from isothermal to adiabatic conditions. The proposed function exhibits a higher accuracy compared to literature formulations.
\end{abstract}

\section{Introduction}

Elevated and high strain rate plastic deformation causes a temperature rise in the material due to a significant fraction of mechanical work being converted to heat. The fraction of work converted into heat is characterized by the Taylor-Quinney coefficient [6], which is often assumed as 0.9 in engineering applications [2]. Under fast loading conditions, heat originating from plastic dissipation is often transferred to the surrounding environment through heat conduction. Accurate modelling of this heat exchange requires transient thermomechanical analyses with complex boundary conditions. In an attempt to obtain meaningful engineering approximations without carrying out a full thermo-mechanical analysis, effective transition functions (so-called $\omega$ functions) have been proposed $[1,3,5]$ to estimate the temperature evolution based on the results from a purely mechanical analysis. These $\omega$ functions introduce a strain rate dependent weighing factor to approximate the transition between the isothermal and adiabatic state.

In this study, uniaxial tensile tests are carried out for a range of strain rates performed on DP800 sheet metal specimens and monitored using infrared imaging. The temperature rise on the specimen surface is measured and the model parameters are identified. A modified logarithmic $\omega$ function is proposed which yields better agreement to experimental data than existing models [1] [3].

* Corresponding author: ccroth@ethz.ch 


\section{Experiments at various loading speeds}

\subsection{Material and specimens}

The material investigated in this project is a sheet metal dual phase steel DP800. All specimens are extracted from $1.6 \mathrm{~mm}$ thick sheets with water-jet cutting. The specimen geometry employed in this work are uniaxial tensile specimens (UT), featuring $20 \mathrm{~mm}$ long and $5 \mathrm{~mm}$ wide gauge sections (Fig. 1).

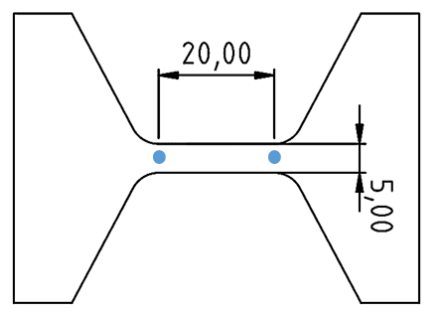

Fig. 1. Specimen geometry for material characterization. The blue dots mark the positions of virtual extensometers for the displacement measurement.

\subsection{Experimental setup}

To investigate the temperature evolution due to plastic heating, UT experiments are carried out at various strain rates. All experiments are performed under displacement control on a $100 \mathrm{kN}$ hydraulic testing machine (Instron, Model 8801). Prior to testing, a random black and white speckle patter is applied on the specimen surface for digital image correlations. The experiments with loading speeds up to $60 \mathrm{~mm} / \mathrm{min}$ are monitored with a $5 \mathrm{MP}$ digital camera (Point Grey GS3-U3-51S5M-C). At higher loading speeds the optical images are recorded with a high-speed camera (Photron SA-Z). With a $105 \mathrm{~mm} \mathrm{1:1} \mathrm{f2.8} \mathrm{macro} \mathrm{lens,} \mathrm{spatial}$ resolutions of $0.0169 \mathrm{~mm} / \mathrm{px}$ and $0.0826 \mathrm{~mm} / \mathrm{px}$, respectively, are obtained. The crosshead displacement rate and their corresponding frame rates are reported in Table 1. To monitor the temperature evolution on the specimen surface, a high-speed infrared camera (FLIR $\mathrm{X} 6801 \mathrm{SC}$ ) is used at a resolution of $640 \times 128$ pixels and the same number of frames per second (fps) as the optical cameras. The image and data acquisition is triggered simultaneously by a rise in the analogue force signal of the load cell. The emissivity of the specimen and the used paint is calibrated by increasing a reference specimen's temperature on a heated plate and comparing thermocouple (type-K) with IR camera measurements.

Table 1. Loading speeds and corresponding frame rates for UT specimens

\begin{tabular}{|c|c|c|c|c|c|c|c|c|c|c|}
\hline $\begin{array}{c}\text { Loading } \\
\text { speeds } \\
{[\mathrm{mm} / \mathrm{min}]}\end{array}$ & 1.2 & 6 & 12 & 60 & 120 & 240 & 600 & 1200 & 2400 & 4800 \\
\hline $\begin{array}{c}\text { Eng. strain } \\
\text { rate }[/ \mathrm{s}]\end{array}$ & $1 \mathrm{E}-3$ & $5 \mathrm{E}-3$ & $1 \mathrm{E}-2$ & $5 \mathrm{E}-2$ & $1 \mathrm{E}-1$ & $2 \mathrm{E}-1$ & $5 \mathrm{E}-1$ & 1 & 2 & 4 \\
\hline $\begin{array}{c}\text { Frame rate } \\
{[\mathrm{Hz}]}\end{array}$ & 1 & 1 & 10 & 50 & 200 & 200 & 500 & 2000 & 2000 & 2000 \\
\hline
\end{tabular}




\section{Results and discussions}

\subsection{Results of the experiments at various strain rates}

Fig. 2a shows the engineering stress-strain curves extracted from the UT tests at various strain rates. The evolution of the surface temperature measured at the center of the gauge section surface (red zone in inset in Fig. 2a) is plotted in Fig. 2b. It is worth noting that for strain rates higher than $0.2 / \mathrm{s}$ the surface temperature evolution converges at around $80^{\circ} \mathrm{C}$. This is an indicator that for strain rates above this value, adiabatic conditions prevail and in numerical simulations solely mechanical analysis with adiabatic conditions can be used.

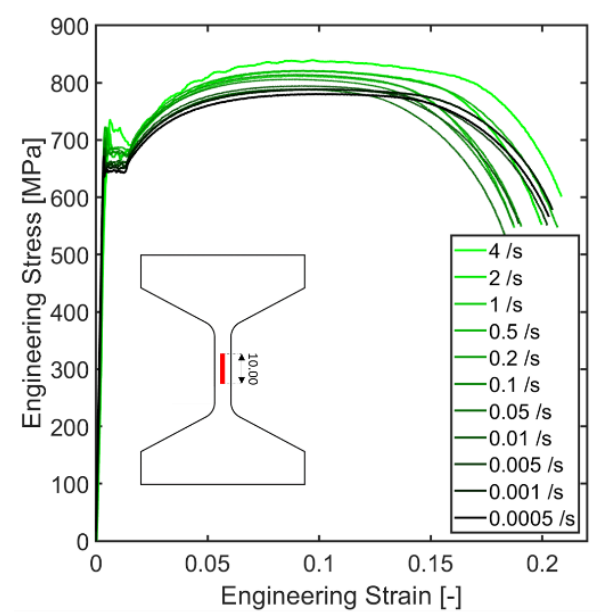

(a)

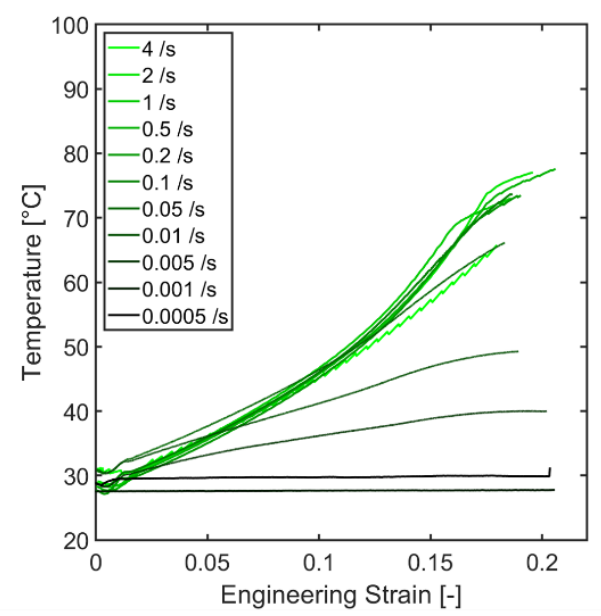

(b)

Fig. 2. (a) Engineering stress-strain curves from uniaxial tensile experiments on DP800 at strain rates from $0.0005 / \mathrm{s}$ to $4 / \mathrm{s}$. The inset of the UT shows the location of the temperature measurement (b) Evolution of specimen surface temperature as a function of engineering strain.

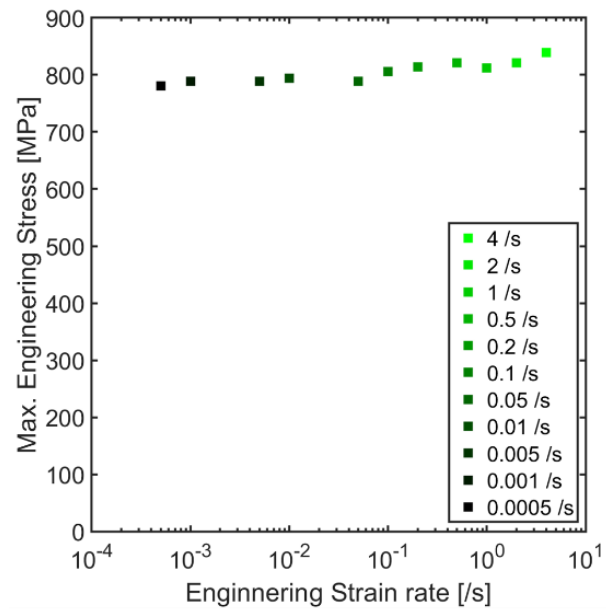

(a)

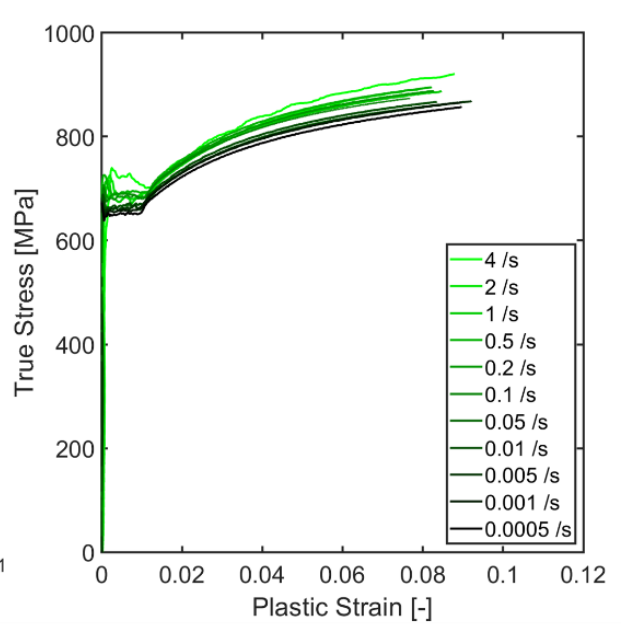

(b)

Fig. 3. (a) UTS plotted as a function of engineering strain rate. Note the monotonic trend of UTS as a function of strain rate. (b) True stress-plastic strain curves from uniaxial tensile experiments on DP800 at various strain rates. 
The maximum engineering stress (UTS) is plotted in Fig. 3a as a function of the engineering strain rate. While negligible deviations exist, overall a monotonic and positive strain rate hardening effect is observed. The corresponding true stress-plastic strain curves are shown in Fig. 3b. These curves are used to evaluate the increments in plastic work up to uniform elongation, which are subsequently used to calculate the percentage of plastic work converted into heat.

\subsection{Estimating the temperature rise}

Neglecting heat exchange with the environment, the incremental temperature rise due to plastic work dissipated as heat is calculated as

$$
d T=\frac{\eta_{k} \bar{\sigma}}{\rho C_{p}} d \bar{\varepsilon}_{p}
$$

where $\eta_{k}$ is the Taylor-Quinney coefficient, $\rho$ is the mass density and $C_{p}$ is the specific heat capacity of the material. This formulation is also often employed in coupled thermomechanical analyses with activated heat conduction.

Alternatively, in an engineering approach, the temperature is treated as an internal state variable in the simulation without modeling the heat exchange between the material point and its surrounding. A first ad-hoc formulation [1] reads

$$
\begin{aligned}
& d T=\omega\left[\dot{\bar{\varepsilon}}_{p}\right] \frac{\eta_{k}}{\rho c_{p}} \bar{\sigma} d \overline{\varepsilon_{p}} \\
& \omega\left[\dot{\bar{\varepsilon}}_{p}\right]_{\mathrm{Roth}}=\left\{\begin{array}{cc}
0 & \dot{\bar{\varepsilon}}_{p}<\dot{\varepsilon}_{i t} \\
\frac{\left(\dot{\bar{\varepsilon}}_{p}-\dot{\varepsilon}_{i t}\right)^{2}\left(3 \dot{\varepsilon}_{a}-2 \dot{\bar{\varepsilon}}_{p}-\dot{\varepsilon}_{i t}\right)}{\left(\dot{\varepsilon}_{a}-\dot{\varepsilon}_{i t}\right)^{3}} & \dot{\varepsilon}_{i t} \leq \dot{\bar{\varepsilon}}_{p} \leq \dot{\varepsilon_{a}} \\
1 & \dot{\varepsilon_{a}}<\dot{\bar{\varepsilon}}_{p}
\end{array}\right.
\end{aligned}
$$

Herein the function $\omega\left[\dot{\overline{\varepsilon_{p}}}\right]$ describes the transition from isothermal to adiabatic conditions. In [1], the isothermal and adiabatic limit strain rates $\varepsilon_{i t}$ and $\dot{\varepsilon}_{a}$ are introduced as the lower and upper bound of the transition zone. Amongst others, Klitschke et al. [3] suggest a twoparameter transition function:

$$
\omega\left[\dot{\bar{\varepsilon}}_{p}\right]_{\text {Klitschke }}=\frac{1}{2}+\frac{1}{\pi} \operatorname{atan}\left[b_{w} \log \left(\frac{\dot{\bar{\varepsilon}}_{p}}{\dot{\bar{\varepsilon}}_{p, w}}\right)\right]
$$

As described in their work, $b_{w}$ and $\dot{\bar{\varepsilon}}_{p, w}$ represent the width and position of the transition zone, respectively.

Experimentally, the fraction of plastic work that leads to an observable temperature rise from infrared camera measurements $\omega\left[\dot{\overline{\varepsilon_{p}}}\right] \cdot \eta_{k}$ is calculated as

$$
\omega\left[\dot{\overline{\varepsilon_{p}}}\right] \eta_{k}=\frac{\bar{\sigma} d \overline{\varepsilon_{p}}}{\rho C_{p} d T}
$$

Figure 4 showcases the performance of a selection of transition functions against the experimental measurements (solid squares with error bars), when choosing the TaylorQuinney coefficient $\eta_{k}$ to 0.82 , the highest inelastic heat fraction obtained experimentally. The transition function of Roth and Mohr [1] (blue dashed line) predicts a much sharper slope than the experimental observations. The function of Klitschke et al. achieves a good correlation for higher strain rates but does not attain zero for lower loading speeds $(0.001 / \mathrm{s})$, which deviates from experimental observations.

Here an alternative transition function based on [1] is proposed to capture the experimentally observed temperature rises:

$$
\omega\left[\dot{\bar{\varepsilon}}_{p}\right]_{n e w}=\left\{\begin{array}{cc}
0, & \dot{\bar{\varepsilon}}_{p}<\dot{\varepsilon}_{i t} \\
\frac{\left(\log \left[\dot{\varepsilon}_{p}\right]-\log \left[\dot{\varepsilon}_{i t}\right]\right)^{2}\left(3 \log \left[\dot{\varepsilon}_{a, \text { new }}\right]-2 \log \left[\dot{\varepsilon}_{p}\right]-\log \left[\dot{\varepsilon}_{i t}\right]\right)}{\left(\log \left[\dot{\varepsilon}_{a, \text { new }}\right]-\log \left[\dot{\varepsilon}_{i t}\right]\right)^{3},} & \dot{\varepsilon}_{i t} \leq \dot{\bar{\varepsilon}}_{p} \leq \dot{\varepsilon_{a}} \\
1, & \dot{\varepsilon_{a}}<\dot{\bar{\varepsilon}}_{p}
\end{array}\right.
$$




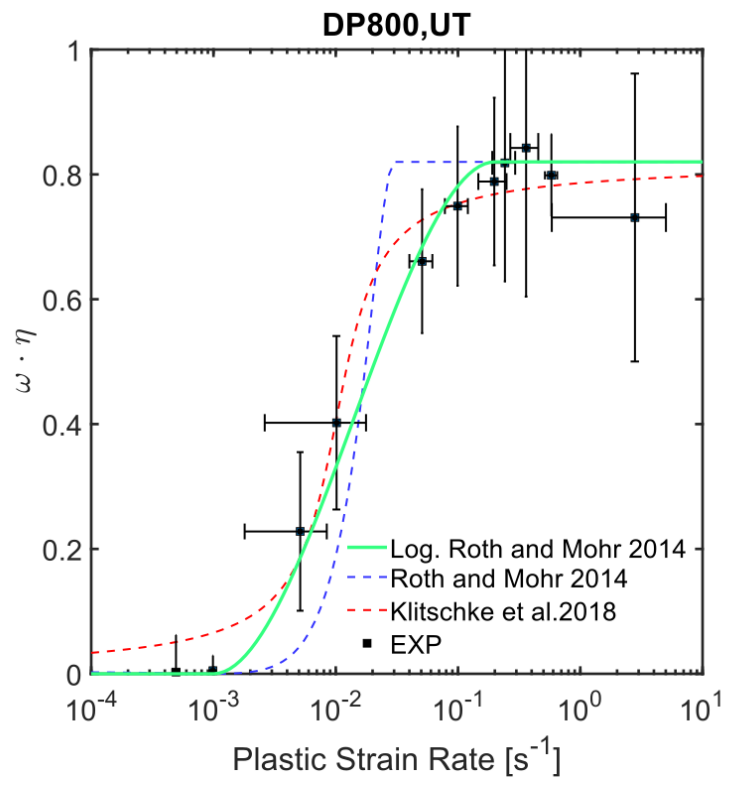

Fig. 4. Transition from isothermal to adiabatic conditions for a range of strain rates. Experimental results are shown as green squares with error bars. The proposed transition function is plotted as a solid green line while the previously proposed functions by [1] and [3] are shown in dashed curves.

The parameters for the transition functions are reported in Table 2. The proposed transition function starts at zero for low strain rates $(<0.001 / \mathrm{s})$ and obtains a very good agreement for the transition phase, as well as the saturation range for strain rates higher $>0.2 / \mathrm{s}$. It should be noted that, as reported by [3], the observed percentage of plastic work converted to heat can change when the material is subjected to loading under different stress states, for example shear loading [4]. This calls for a thorough experimental and numerical investigation of the dependency of the "compound" Taylor-Quinney coefficient $\omega \cdot \eta_{k}$ on the stress state, the strain, the strain rate and possibly the temperature.

Table 1. Model parameters for DP780

\begin{tabular}{cccccc}
\hline Material Constants & $E$ & $v$ & $\rho$ & $C_{p}$ & $\eta_{k}$ \\
& {$[\mathrm{MPa}]$} & {$[-]$} & {$\left[\mathrm{kg} / \mathrm{m}^{3}\right]$} & {$[\mathrm{J} / \mathrm{kgK}]$} & {$[-]$} \\
\hline & 210 & 0.3 & 7850 & 454 & 0.82 \\
\hline \hline Roth and Mohr [1] & $\dot{\varepsilon_{i t}}$ & $\dot{\varepsilon_{a}}$ & & & \\
& {$[1 / \mathrm{s}]$} & {$[1 / \mathrm{s}]$} & & & \\
\hline & 0.001 & 0.03 & & \\
\hline \hline Klitschke et al. [3] & $b_{w}$ & $\dot{\bar{\varepsilon}}_{p, w}$ & & \\
& {$[-]$} & {$[1 / \mathrm{s}]$} & & \\
\hline & 1.674 & 0.01 & & \\
\hline \hline Log. Roth and Mohr & $\dot{\varepsilon}_{i t}$ & $\dot{\varepsilon}_{a, n e w}$ & & \\
(this work) & {$[1 / \mathrm{s}]$} & {$[1 / \mathrm{s}]$} & & & \\
\hline & 0.001 & 0.2 & & \\
\hline \hline
\end{tabular}




\section{Conclusion}

In this work a series of uniaxial tensile tests on DP800 sheet metal specimens are performed at strain rates from $0.001 / \mathrm{s}$ to $4 / \mathrm{s}$. A monotonic effect of the strain rate on the hardening response is observed. The temperature evolution converges for strain rates above $0.2 / \mathrm{s}$, indicating adiabatic conditions for loading speeds above this strain rate. A modified logarithmic based function is proposed to model the transition from isothermal to adiabatic conditions. The proposed function achieves better agreement with the experimental results when compared to previously proposed functions $[1,3]$.

\section{References}

1. C. C. Roth and D. Mohr, Effect of strain rate on ductile fracture initiation in advanced high strength steel sheets: Experiments and modelling, International Journal of Plasticity, vol. 56, pp. 19-44 (2014)

2. J. Hodowany, G. Ravichandran, A. J. Rosakis, and P. Rosakis, "Partition of plastic work into heat and stored energy in metals," Exp. Mech., vol. 40, no. 2, pp. 113-123, 2000

3. S. Klitschke, A. Trondl, F. Huberth, and M. Liewald, "Adiabatic heating under various loading situations and strain rates for advanced high-strength steels," IOP Conf. Ser. Mater. Sci. Eng., vol. 418, no. 1, 2018

4. X. Li, C. C. Roth, T. Tancogne-Dejean, and D. Mohr, "Rate- and temperaturedependent plasticity of additively manufactured stainless steel 316L: Characterization, modeling and application to crushing of shell-lattices," Int. J. Impact Eng., vol. 145, no. July, p. 103671, 2020

5. D. Rittel, L. H. Zhang, and S. Osovski, "The dependence of the Taylor-Quinney coefficient on the dynamic loading mode," J. Mech. Phys. Solids, vol. 107, pp. 96-114, 2017

6. G. I. Taylor and H. Quinney, "The latent energy remaining in a metal after cold working," Proc. R. Soc. London. Ser. A, Contain. Pap. a Math. Phys. Character, vol. 143, no. 849 , pp. $307-326,1934$ 